\title{
EVALUACIÓN DE LA LIXIVIACIÓN DE MERCURIO EN RESIDUOS SÓLIDOS peligrosos generados en industrias de Cloro-Sosa
}

\author{
EVALUATION OF THE LEACHING OF MERCURY IN HAZARDOUS SOLID \\ WASTE GENERATED BY INDUSTRIES CHLORINE-CAUSTIC
}

Ph.D. Yailen Busto Yera Universidad Israel - Ecuador

ybusto@hotmail.com

.Fecha de recepción: 01/03/2015

Fecha de aceptación: 28/03/2015

\begin{abstract}
Resumen
A través del proceso de producción convencional de cloro-álcali, se producen elevadas cantidades de lodos residuales contaminados con mercurio ( $\mathrm{Hg}$ ). La inapropiada manipulación y disposición de estos lodos puede causar un peligro ambiental. El comportamiento de lixiviación del $\mathrm{Hg}$ presente en estos lodos mercuriales, procedentes de una planta de cloro-álcali que todavía está en funcionamiento, se investigó mediante la prueba alemán DIN 38414-S4. El contenido de mercurio total de las muestras se mostró por encima de $1500 \mathrm{mg} / \mathrm{kg}$, permitiendo clasificar el material como residuo peligroso y de alto mercurio.

Las concentraciones de $\mathrm{Hg}$ en los lixiviados para ambas muestras fueron superiores a 0,02 mg/1, valor establecido por la Directiva de la Comunidad Económica Europea (CEE) en 1991 sobre disposición final en vertederos , como límite máximo de mercurio para su disposición en vertederos. Las altas concentraciones de la muestra 2 sugirieron la presencia de especies más solubles de mercurio, tales como el $\mathrm{HgC12}$. Los resultados indican que el método utilizado para estabilizar el lodo en dicha industria, no ha sido suficientemente eficaz, y
\end{abstract}


justifican la precaución sobre los sitios de disposición existentes así como la gestión futura de estos residuos mercuriales altamente peligrosos.

Palabras claves: Mercurio, Residuos mercuriales peligrosos, Movilidad del mercurio, Prueba de lixiviación.

\section{Abstract}

Through the conventional chlor-alkali production process, highly mercury $(\mathrm{Hg})$ contaminated waste sludges are produced. Improper handling and disposal of this sludge may cause an environmental hazard. The leaching behavior of $\mathrm{Hg}$ of land disposed mercurial sludge, originating from a chlor-alkali plant that still is in operation, was investigated using the German DIN 38414-S4 test. The total mercury content of the samples was above $1500 \mathrm{mg} / \mathrm{kg}$, allowing the material to be classified as hazardous and high mercury waste. Concentrations of $\mathrm{Hg}$ in the leachates for both samples were higher than $0.02 \mathrm{mg} / 1$ stipulated by the 1991 EEC Landfill Directive Draft as a maximum limit for a waste that is to be landfilled.

The high concentrations of Sample 2 suggested the presence of rather soluble mercury species such as $\mathrm{HgC} 12$. Results indicate that the approach used to stabilize the sludge has not been sufficiently effective, and warrant caution about existing disposal sites and future management of these mercury containing wastes.

Keywords: Mercury, Hazardous mercurial wastes, Mercury mobility, Leaching test. 


\section{Introducción}

Las plantas de cloro-álcali con celdas de mercurio (MCCAP) producen cloro y sosa cáustica por electrólisis de salmuera, utilizando mercurio como cátodo. En este proceso, son manejadas grandes cantidades de mercurio y debido a la alta presión de vapor de mercurio a bajas temperaturas, considerables cantidades de mercurio son emitidas a la atmósfera, a causa de derrames o fugas en las celdas de mercurio y otras actividades (Southworth G. R., 2004).

Aunque este proceso se basa en la tecnología del siglo XIX, MCCaPs en todo el mundo, todavía representan aproximadamente el $15 \%$ de la demanda mundial de mercurio, y son una fuente importante de contaminación por mercurio local y global (Biester H., 2002); (Landis M. S., 2004); (Ulrich S., 2007); (K., 2009); (Reis A. T., 2009).

Estimaciones más recientes indican que las emisiones de la industria del cloro-álcali son responsables de cerca del 17\% o 40,4 t/año de emisiones antropogénicas de mercurio (Pacyna E. G., 2006). Estos procesos no se consideran ya una buena práctica industrial. El comité de prevención integrada y control de la contaminación (IPPC) de la Unión Europea ha indicado que las instalaciones de cloro-álcali requieren la obtención de licencias basadas en las mejores técnicas disponibles (200811/ EC , 2008). La tecnología para la producción de cloro y sosa cáustica base mercurio debe ser sustituida por otras tecnologías que son más compatibles con el medio ambiente, tales como las que implican el uso de diafragma y celdas de membrana.

En las últimas décadas, los procesos celdas de membrana y diafragma han sustituido a la tecnología de celdas de Hg en Europa, Estados Unidos y Japón (Mukherjee A. B., 2004). Muchos propietarios de plantas en Europa y EE.UU. ya han anunciado planes para el desmantelamiento antes del 2020.

Sólo alrededor de 50 plantas no han anunciado sus planes o no están obligadas a desmantelar en 2020 debido a que su producción combinada es bastante pequeña, por lo que la conversión de estas plantas restantes es factible. Según los datos recopilados por el PNUMA durante el inventario mundial de celdas de mercurio en instalaciones de cloro-álcali (PNUMA, 2009), 100 instalaciones en 44 países hoy tienen producción de cloro industrial empleando tecnología de cátodo de mercurio. Sin embargo, sólo 12 países cuentan con un plan de cierre o conversión a tecnologías sin mercurio durante el período 2010 a 2015 (Tabla 1). 
Tabla 1. Los países con las más altas capacidades de producción de cloro empleando celdas de mercurio

\begin{tabular}{|c|c|c|c|}
\hline País & $\begin{array}{l}\text { Plantas de } \\
\text { celdas de } \\
\text { mercurio }\end{array}$ & $\begin{array}{l}\text { Capacidad de } \\
\text { producción de } \\
\text { claro año } 2010^{\mathrm{a}}\end{array}$ & $\begin{array}{l}\text { Planes para cierre o conversión a tecnología sin mercurio } \\
\qquad 2010-2015\end{array}$ \\
\hline Alemania & 6 & 870 & $\begin{array}{l}\text { - Ludwigshafen (BASF) no especifica la fecha de conver- } \\
\text { sión o cierre en el permiso. } \\
\text { - Akzo Nobel (Ibbenburen), la conversión o cierre estiman } \\
\text { entre 2010-2015. } \\
\text { - Fecha de conversión o cierre de Evonic (Degussa) no se } \\
\text { especifica en el permiso. } \\
\text { - Akzo Nobel (Frankfurt), conversión estimada para el año } \\
2010 \text {. }\end{array}$ \\
\hline España & 7 & 732 & $\begin{array}{l}\text { Todas las plantas de España tienen que submitir su plan de } \\
\text { cierre o conversión para el } 2011 \text {. }\end{array}$ \\
\hline Francia & 6 & 690 & $\begin{array}{l}\text { - Planta Jarrien espera su conversión tecnológica en el } 2012 . \\
\text { - Solvay Tavaux - } 2 \text { plantas están fuera de las } 4 \text { unidades } \\
\text { que emplean mercurio, una planta se convirtió en el } 2010 .\end{array}$ \\
\hline Estados Unidos & 4 & 437 & \\
\hline Bélgica & 2 & 420 & $\begin{array}{l}\text { Las plantas Tessenderlo y Antwerp planean cambiar a tecnolo- } \\
\text { gía de membrana en el } 2015 \text { y } 2012 \text { respectivamente. }\end{array}$ \\
\hline Rusia & 3 & 401 & \\
\hline Irán & 4 & 332 & \\
\hline Reino Unido & 1 & 277 & \\
\hline Brasil & 4 & 217 & \\
\hline $\mathrm{Cuba}^{\mathrm{b}}$ & 1 & 7 & \\
\hline
\end{tabular}

Fuente: creado por la autora 
En Cuba, la producción de cloro y sosa cáustica se inició a mediados de los años 30 en la ciudad de Sagua La Grande, situada en el centro del país. La planta tiene una capacidad de producción por día de 48 toneladas de cloro gaseoso y 108 toneladas de sosa cáustica en una concentración del $50 \%$.

En la actualidad, la Electroquímica "Elpidio Sosa", la única industria de este tipo en el país, cubre la demanda interna de estos productos y el excedente se exporta a la región del Caribe. En el 2008, el gobierno cubano aprobó los planes para un cambio en la tecnología de celdas de mercurio a la tecnología basada en membranas. Si bien esta transición evitaría una mayor producción de residuos contaminados con mercurio, miles de toneladas de lodos mercuriales se almacenan actualmente en los alrededores de la planta, lo que constituye un posible riesgo para la salud humana y el medio ambiente si es que no se toman acciones para su adecuado tratamiento.

En la evaluación de los riesgos ambientales, las pruebas de lixiviación pueden proporcionar información sobre la movilidad/disponibilidad esperada de los metales peligrosos a través del agua y aguas subterráneas, el suelo, los sedimentos y los residuos sólidos. En la literatura, dos tipos de pruebas de lixiviación se distinguen para determinar la movilidad de los metales pesados en las muestras: pruebas individuales por lotes/dinámicas y pruebas de extracción múltiple/flujo continuo (R. C. V., 2008) .

Las pruebas individuales de extracción por lotes típicamente implican mezclar una muestra con una cantidad específica de la solución de lixiviación sin renovación de la misma (Ecology, 2003) (Bayar S., 2009). En ensayos de lixiviación dinámica, donde el tiempo es también una variable importante, una cantidad específica de la solución de lixiviación y material de ensayo se mezclan y la solución de lixiviación se renueva periódicamente o de forma continua. La mezcla se realiza en un período relativamente largo de tiempo (días o meses), en comparación con las pruebas de extracción de lotes individuales (Fytianos K ., 1998).

El ensayo de lixiviación DIN 38414-S4 es un método de lixiviación dinámica para la evaluación del impacto ambiental. Ha sido desarrollado por el Instituto Alemán de Normalización (DIN) en 1984. Se utiliza habitualmente para evaluar la lixiviación de metales para su disposición final en vertederos (Viguri J., 2000); (Kylefors K., 2003); (Kazi T. G., 2005); (Gonzalez-Fernandez 0., 2009). Según (Quevauviller P. H., 1996) la prueba DIN es un método simple para evaluar aquella fracción de mercurio más débilmente unida a la matriz sólida, que sea fácilmente movilizable o biodisponible en las muestras de residuos sólidos o suelo contaminado.

El límite permisible de mercurio en el líquido lixiviado es de 0,02 mg/l de acuerdo con la legislación de 1991 de la Comunidad Económica Europea (CEE) en su Directiva sobre disposición en vertederos (Bayar S., 2009).

Los residuos sólidos conteniendo mercurio son generados en la industria cubana después de mezclar el mercurio agotado de la celda electrolítica con sulfuro de sodio en un primer paso, y con cloruro de sodio, carbonato de calcio, hidróxido de magnesio y la tierra de diatomeas en un segundo paso. Actualmente, este lodo mercurial constituye un riesgo ambiental pues está dispuesto en nichos de hormigón. El contacto con el agua subterránea o la lluvia puede hacer que el mercurio se filtre y contamine el suelo y las aguas subterráneas. Estudios sobre el comportamiento de lixiviación de los lodos mercuriales generados en la industria de cloro-álcali no están 
disponibles. En el presente trabajo, se utilizó la prueba de lixiviación DIN-S4 para evaluar las liberaciones de mercurio a partir de este lodo contaminado. El objetivo principal de este estudio es evaluar el riesgo ambiental real que este lodo mercurial representa para el hombre y el medio ambiente.

\section{Materiales y métodos}

\section{Descripción del sitio}

Las muestras de lodo mercurial se tomaron de un sitio cerca de la planta electroquímica que produce cloro y sosa, ubicada al norte de la región central de Cuba. El lodo mercurial generado por el proceso electrolítico está enterrado en nichos de concreto dispersos alrededor de la fábrica. Los nichos son de 9,5 m de largo, 4,5 m de ancho y 3,5 m de alto. Los mismos, permiten el almacenamiento de $150 \mathrm{~m}^{3}$ de lodo mercurial.

\section{Muestreo y preparación de las muestras}

Dos muestras fueron recolectadas en diferentes nichos. La muestra 1 ha estado un año y cuatro meses en el nicho, mientras que la muestra 2 ha tenido un tiempo de residencia de sólo cuatro meses en el nicho antes del muestreo. Cada muestra se preparó como una muestra compuesta de cantidades iguales de los lodos extraídos de tres puntos diferentes en una línea diagonal, a $20 \mathrm{~cm}$ de los dos extremos de los nichos y en el punto central, así como a tres profundidades: 0-20, 20-40 y 40-60 cm (C ., 2004). Inmediatamente después de su llegada al laboratorio, las muestras se secaron al aire durante siete días, trituradas a mano en un mortero, pasadas por un tamiz de 2 mm y mezcladas completamente.

\section{Caracterización de las muestras}

El pH de los residuos sólidos se midió después de 18 horas utilizando un medidor de pH modelo Orion 520 (Orion, Boston, MA, EE.UU.) en una suspensión de $50 \mathrm{ml}$ de agua destilada con $10 \mathrm{~g}$ de lodos mercuriales (Cottenie A ., 1982). La materia orgánica se estimó pesando $1 \mathrm{~g}$ de la muestra antes y después de calcinación a $400{ }^{\circ} \mathrm{C}$ durante 2 horas (Van Ranst E., 1999). El contenido de carbonato se determinó por titración en retroceso de un exceso de 0,50M de H2S04 adicionando 0,25 g de la muestra con 0,5 M de NaOH (E . N. R., 1982). La capacidad de intercambio catiónico (CIC) a pH neutro se determinó por el método de acetato de amonio (S., 1996) utilizando $5 \mathrm{~g}$ de la muestra de lodo mercurial.

Para determinar el contenido de cloruro, $1 \mathrm{~g}$. de muestra de lodo se suspendió en $50 \mathrm{ml}$. de 0,15mol/1 de HNO3 y se agitó durante 30 minutos. El filtrado se valoró con 0,05mol/1 de AgN03 con punto final de detección potenciométrica (Van Ranst E., 1999) usando un potenciómetro Metrohm 761 Compact IC (Metrohm, Herisau, Suiza). 
El contenido total de mercurio se determinó mediante la técnica de espectrofotometría de absorción atómica por vapores fríos (Mercury Analyzer MAS-50, Coleman, Oak Brook, Illinois, EE.UU.), después de una destrucción específica según lo reportado por (Cottenie A., 1982). El procedimiento de destrucción implicó la adición de $0,05 \mathrm{~g}$ de V205, y $10 \mathrm{ml}$ de HN03 concentrado a $0,5 \mathrm{~g}$ de muestra, con una digestión de 30 minutos a $160^{\circ} \mathrm{C}$. Después de que las muestras se enfriaron se añadieron $15 \mathrm{ml}$. de H2S04 concentrado y la digestión se completó durante 2 horas sin calentamiento adicional.

Una solución estándar de 0,25 mg/l de mercurio se preparó a partir una solución patrón de $1000 \mathrm{mg} / 1$ (Solución Estándar Mercury, Merck, Darmstadt, Alemania). La curva de calibración se obtuvo midiendo la transmitancia después de la inyección de diferentes volúmenes $(0,0,5,1,2,4$ y $8 \mathrm{ml})$. El contenido pseudo-total de metales se determinó después de la destrucción en agua regia (M., 1990), utilizando la técnica de Espectrometría de emisión óptica con plasma acoplado inductivamente (ICP-OES, Vista MPX CCD simultánea, Varian, Mulgrave VIC, Australia). Cada determinación se realizó por triplicado y todos los reactivos utilizados fueron de grado analítico.

\section{Prueba de Lixiviación DIN384 14 -54}

El nivel de lixiviación de $\mathrm{Hg}$ presente en el lodo mercurial se evaluó mediante el ensayo de lixiviación DIN38414-S4 (38414-S4, 1984). La razón líquido a sólido (LIS) se estableció como la relación másica entre el agente lixiviante y la muestra de lodo durante la lixiviación. La relación LIS acumu lativa osciló desde 10 hasta 40.

$5 \mathrm{~g}$. de residuos sólidos se transfirieron a un tubo de centrifugado (volumen $100 \mathrm{ml}$ ) y se suspendieron en $50 \mathrm{ml}$. de agua destilada como agente de lixiviación, correspondiendo con una relación LIS de 10. Las suspensiones se agitaron de forma continua utilizando un agitador mecánico del tipo "end over end" a $30 \mathrm{rpm}$.

Después de 24 horas, se centrifugaron las muestras (Megafuge 1.0, Heraeus Instruments GmbH, Heraeus, Alemania), durante 12 minutos a $3000 \mathrm{rpm}$ para permitir la sedimentación de los materiales suspendidos. La solución sobrenadante se decantó y se filtró empleando un filtro de membrana de tamaño de poro de 0,45 micras (CM, Porafil, Macherey-Nagel, Düren, Alemania). Se añadió agente de lixiviación fresco, y el procedimiento de extracción se repitió hasta que se tuvieron las 4 fracciones.

Después de la determinación del pH y la conductividad eléctrica de la muestra de lixiviado, se acidificó a un pH por debajo de 2 con HN03 concentrado para almacenar la muestra antes del análisis de mercurio. Todas las extracciones se llevaron a cabo por triplicado. La conductividad eléctrica se midió en los extractos utilizando un microprocesador medidor de conductividad (LF 537, Weilhem, Alemania).

El pH se determinó potenciométricamente usando un pH-metro modelo Orion 520 (Orion, Boston, MA, EE.UU.). La medición de los metales Ca y Mg se realizó mediante ICP-OES (Vista MPX CCD simultánea, 
Varian, Mulgrave VIC, Australia). Por otra parte la técnica de fotometría de llama (ELEX 6361, Eppendorf, Hamburgo, Alemania) se utilizó para determinar la concentración de los metales Na y K.

El contenido total de mercurio en los lixiviados se determinó empleando la técnica de espectrofotometría de absorción atómica por vapores fríos (Mercury Analyzer MAS-50, Coleman, Oak Brook, Illinois, EE.UU.). Para el análisis, una alícuota de $20 \mathrm{ml}$ de solución de la muestra, $10 \mathrm{ml}$ de HN03 concentrado y $15 \mathrm{ml}$ de H2S04 concentrado se añadieron en $250 \mathrm{ml}$ de matraz volumétrico y se diluyó a ese volumen con agua desionizada. La calibración se realizó como se ha explicado antes. El límite de detección (LDD), definido como tres veces la desviación estándar de las muestras de partida fue de 0,02 mg/l. Todos los análisis se llevaron a cabo por triplicado.

\section{Resultados}

\section{Características químicas del residual sólido}

El lodo mercurial exhibió un pH alcalino de 9.3 a 9.4 (Tabla 2). Esto es causado por la presencia de carbonato de calcio e hidróxido de magnesio, dos componentes añadidos durante la estabilización del producto residual.

Tabla 2 . Características químicas del lodo mercurial

\begin{tabular}{|l|l|l|}
\hline Parámetros & Muestra 1 & Muestra 2 \\
\hline $\mathrm{pH}-\mathrm{H}_{2} \mathrm{O}$ & $9.3(0,03)$ & $9.4(0,03)$ \\
\hline Materia orgánica & $158(5,1)$ & $102(5.8)$ \\
\hline $\mathrm{CaCO}_{3}(\mathrm{~g} / \mathrm{kg})$ & $667(5,8)$ & $733(5,8)$ \\
\hline Cloruros $(\mathrm{g} / \mathrm{kg})$ & $57.2(0,4)$ & $60.5(1,8)$ \\
\hline $\mathrm{CIC}^{\mathrm{b}}(\mathrm{cmol} / \mathrm{kg})$ & $11.8(0,4)$ & $7.8(0,3)$ \\
\hline $\begin{array}{l}\text { Valores entre paréntesis representan la desviación estándar de tres réplicas. } \\
\text { Capacidad de intercambio catatónico. }\end{array}$ & \\
\hline
\end{tabular}

Fuente: autora.

El contenido de carbonato estaba en el orden de 60-70\%, mientras que el análisis de metales pseudo totales reveló altas concentraciones de $\mathrm{Ca}$ y $\mathrm{Mg}$ (Tabla 3). Aproximadamente $300 \mathrm{~g}$. de tierra de diatomeas se utiliza 
para la producción de $1 \mathrm{~kg}$. de lodo mercurial, por lo que también contribuye en el nivel de carbonato en el producto final de residuos.

Tabla 3. Contenido pseudo-total de metales en el lodo mercurial.

\begin{tabular}{|l|l|l|}
\hline \multicolumn{1}{|c|}{ Contenido de metales } & \multicolumn{1}{c|}{ Muestra 1 } & \multicolumn{1}{c|}{ Muestra 2 } \\
\hline $\mathrm{Ca}(\mathrm{g} / \mathrm{kg})$ & $223(1)$ & $275(3)$ \\
\hline $\mathrm{Mg}(\mathrm{g} / \mathrm{kg})$ & $32(0,2)$ & $18(0,2)$ \\
\hline $\mathrm{Fe}(\mathrm{g} / \mathrm{kg})$ & $10(0,1)$ & $10(0,4)$ \\
\hline $\mathrm{Hg}(\mathrm{g} / \mathrm{kg})$ & $5.6(0,2)$ & $1.5(0,1)$ \\
\hline $\mathrm{Pb}(\mathrm{mg} / \mathrm{kg})$ & $44(1,4)$ & $58(4,4)$ \\
\hline $\mathrm{Zn}(\mathrm{mg} / \mathrm{kg})$ & $177(2)$ & $181(0,5)$ \\
\hline $\mathrm{Ni}(\mathrm{mg} / \mathrm{kg})$ & $31(0,3)$ & $23(1)$ \\
\hline $\mathrm{Cu}(\mathrm{mg} / \mathrm{kg})$ & $169(1)$ & $455(4)$ \\
\hline $\mathrm{Cr}(\mathrm{mg} / \mathrm{kg})$ & $40(0,4)$ & $27(1)$ \\
\hline $\mathrm{Cd}(\mathrm{mg} / \mathrm{kg})$ & $1.8(0,1)$ & $2.6(0,1)$ \\
\hline $\mathrm{Mn}(\mathrm{mg} / \mathrm{kg})$ & $235(1)$ & $232(2)$ \\
\hline $\mathrm{Valores} \mathrm{entre} \mathrm{paréntesis} \mathrm{representan} \mathrm{la} \mathrm{desviación} \mathrm{estándar} \mathrm{de} \mathrm{tres} \mathrm{réplicas.}$ & \\
\hline
\end{tabular}

Fuente: autora.

La tierra de diatomeas también contiene alrededor de $6 \%(\mathrm{w} / \mathrm{w})$ de la materia orgánica y aproximadamente $2 \%$ (w/w) de Fe (E. A. L., 1998). Esto explica las concentraciones relativamente altas de hierro (Fe) en el lodo mercurial. Niveles elevados de cloruro, en el orden de $50 \mathrm{~g} / \mathrm{kg}$ se encontraron, lo que indica la presencia de una cantidad sustancial de sales solubles.

Entre las muestras, los contenidos totales de mercurio difieren por un factor de tres, que apuntan a una marcada variabilidad en la producción de dichos lodos mercuriales. En ambas muestras, las concentraciones de mercurio fueron muy superiores a $260 \mathrm{mg} \mathrm{Hg} / \mathrm{kg}$ de lodo, por lo que los materiales se clasifican como residuos 
peligrosos y de alto contenido de mercurio de acuerdo a la EPA de EE.UU. (USEPA , 2008). Otros elementos traza potencialmente tóxicos se encontraron en niveles superiores a los rangos normales presentes en los suelos (H., 1984).

\section{Lixiviación real}

El ensayo de lixiviación DIN S4 proporciona indicaciones sobre la liberación de los elementos cuando se pone en contacto el material contaminado con cantidades crecientes de agente de lixiviación.

Los altos valores de $\mathrm{pH}$ de los lixiviados (Tabla 4) son resultado de la presencia de hidróxidos libres proporcionados por el $\mathrm{Mg}(\mathrm{OH}) 2$ utilizado durante la preparación de los lodos. Para las dos muestras el pH aumentó aún más en la segunda fracción. Este aumento puede reflejar un efecto cinético, donde los lodos inicialmente secos interactúan más lento con el agente de lixiviación añadido que el lodo húmedo en las fases de extracción subsiguientes. Los valores altos de conductividad eléctrica en la primera fracción revelan la presencia de cantidades significativas de sales solubles. Este lixiviado se clasifica como altamente salino según la FAO (Rhoades J., 1992).

Tabla 4 . pH y conductividad eléctrica en fracciones de la prueba de lixiviación DIN384 14-S4a

\begin{tabular}{|c|c|c|c|c|}
\hline \multirow{3}{*}{ Muestras } & \multicolumn{4}{|c|}{ Razón L/S } \\
\hline & 10 & 20 & 30 & 40 \\
\hline & \multicolumn{4}{|c|}{ pH } \\
\hline 1 & $9.49(0,06)$ & $9.92(0,05)$ & $9.97(0,03)$ & $9.97(0,01)$ \\
\hline 2 & $9.51(0,03)$ & $9.84(0,05)$ & $9.8(0,05)$ & $9.65(0,02)$ \\
\hline \multicolumn{5}{|c|}{ Conductividad eléctrica $(\mathrm{mS} / \mathrm{cm})$} \\
\hline 1 & $19.1(0,2)$ & $2.42(0,04)$ & $0.58(0,01)$ & $0.25(0)$ \\
\hline 2 & $19.7(0,11)$ & $2,19(0,02)$ & $0.48(0)$ & $0.18(0)$ \\
\hline
\end{tabular}

Fuente: autora. 
Esto puede ser principalmente $\mathrm{NaCl}$ según lo sugerido por los niveles muy altos de $\mathrm{Na}$ en comparación con los otros cationes presentes en los extractos (Tabla 5, tenga en cuenta que Na está en g/l). El sodio se utiliza en diferentes puntos del proceso de producción de cloro-álcali, como materia prima $(\mathrm{NaCl})$ en el proceso de celda electrolítica y como Na2S añadido al lodo residual para estabilizar el $\mathrm{Hg}$ en el lodo. La rápida disminución de la conductividad eléctrica con el aumento de relación L/S acumulada, indica la eliminación de las sales solubles durante los pasos consecutivos de la prueba de lixiviación. A diferencia del Na, el K y en menor medida del $\mathrm{Mg}$, las concentraciones de Ca fueron más constantes en las fracciones posteriores (Tabla 5). Esta observación es consistente con la limitada solubilidad de los carbonatos de calcio.

Tabla 5 . Concentración de metales en las fracciones de la prueba de lixiviación DIN38414-S4a

\begin{tabular}{|c|c|c|c|c|}
\hline Razón L/S & 10 & 20 & 30 & 40 \\
\hline \multicolumn{5}{|c|}{ Muestra 1} \\
\hline $\mathrm{Na}(\mathrm{g} / \mathrm{l})$ & $3.75(0,09)$ & $0.44(0,01)$ & $0.092(0,02)$ & $0.041(0,01)$ \\
\hline $\mathrm{K}(\mathrm{mg} / \mathrm{l})$ & $23.6(0,87)$ & $3.8(0)$ & $1.73(0,23)$ & $1.13(0,12)$ \\
\hline $\mathrm{Ca}(\mathrm{mg} / \mathrm{l})$ & $2.41(0,18)$ & $1.61(0,07)$ & $1.04(0,04)$ & $1.19(0,04)$ \\
\hline $\operatorname{Mg}(\mathrm{mg} / \mathrm{l})$ & $10.2(1,76)$ & $0.93(0,12)$ & $0.27(0)$ & $0,29(0,05)$ \\
\hline \multicolumn{5}{|c|}{ Muestra 2} \\
\hline $\mathrm{Na}(\mathrm{g} / \mathrm{l})$ & $4.05(0,12)$ & $0.4(0,003)$ & $0.074(0)$ & $0.031(0,004)$ \\
\hline $\mathrm{K}(\mathrm{mg} / \mathrm{l})$ & $9.67(2,67)$ & $1.67(0,23)$ & $0.73(0,23)$ & $0.67(0,12)$ \\
\hline $\mathrm{Ca}(\mathrm{mg} / \mathrm{l})$ & $2.51(0,05)$ & $1.65(0,03)$ & $1.2(0,05)$ & $1.66(0,09)$ \\
\hline $\operatorname{Mg}(\mathrm{mg} / \mathrm{l})$ & $8.56(0,54)$ & $0.71(0,03)$ & $0.27(0)$ & $0.4(0,01)$ \\
\hline
\end{tabular}

Fuente: autora.

Las concentraciones de $\mathrm{Hg}$ en las fracciones de la prueba DIN difieren en gran medida, por más de un orden de magnitud, en tre las dos muestras (Figura 1). En general, las concentraciones de Hg disminuyeron en frac- 
ciones posteriores de la prueba de lixiviación DIN, aunque la muestra 2 exhibió un aumento pronunciado, por un factor de tres, en la concentración de mercurio en·la segunda fracción en comparación con el primero. Es difícil explicar el diferente comportamiento entre los lodos teniendo en cuenta que las características químicas de ambos lodos verificadas experimentalmente fueron similares (Tabla 2).

Como la muestra 1 es un año mayor, estuvo más limitada la liberación de $\mathrm{Hg}$ en esa muestra, a pesar de su mayor contenido de $\mathrm{Hg}$ total (Tabla 3), esto puede atribuirse a un efecto de envejecimiento. Sin embargo, también podría revelar una variabilidad significativa en la calidad de la estabilización aplicada a los lodos mercuriales.

Un mues treo y análisis de los lodos producidos regularmente sería necesario para determinar la variabilidad en la producción. La lixiviación acumulada que mostró el mercurio en las muestras puede ser descrita por una ecuación logarítmica, según:

$$
\begin{gathered}
C M R_{1}=2.00 \ln (L / S)-2.97 \\
C M R_{2}=64.2 \ln (L I S)-129
\end{gathered}
$$

Donde: CMR representan el mercurio acumulado en la lixiviación en $\mathrm{mg} / \mathrm{kg}$ de lodo seco, y L/S representa la relación líquido/sólido. Los subíndices representan los números de la muestra. Los valores de R2 de las ecuaciones 1 y 2 fueron de 0,995 y 0,9904 respectivamente. Ecuaciones similares fueron reportadas por (R. C. V., 2008) para describir la lixiviación del mercurio y otros metales en suelos contaminados.

La pendiente de cada ecuación se puede considerar como una medida de la intensidad de la lixiviación de $\mathrm{Hg}$, y representa una ten dencia del $\mathrm{Hg}$ para ser liberado de la matriz de lodos. La lixiviación acumulada de $\mathrm{Hg}$ durante todo el ensayo. de lixiviación ascen dió a menos de $0,1 \%$ para la muestra 1, pero estuvo en el orden de 7,0\% para la muestra 2, que arrojó un contenido total de $\mathrm{Hg}$ inferior pero una lixiviación mucho más intensiva.

La Directiva del Consejo 91/689/CEE, relativa a los residuos peligrosos es ampliamente utilizada para la caracterización de los residuos antes de su vertido al medio ambiente. La Directiva establece cuándo un residuo puede ser considerado un "desecho peligroso". Asímismo, define los valores de concentración límite para cada constituyente peligroso y los métodos de prueba aplicables $(91 / 689 / \mathrm{EEC}, 1991)$.

De acuerdo con esta directiva, la prueba DIN38414-S4 se utiliza normalmente para caracterizar el comportamiento de lixiviación de compuestos en la muestra. El valor límite para la concentración de mercurio en el lixiviado es de 0,02 mg/l (Bayar S., 2009).

Las concentraciones de mercurio en todas las fracciones de la muestra 2, por mucho, superan este límite permisible (Figura 1). Aunque la muestra 1 mostró una liberación mucho más lenta de $\mathrm{Hg}$, los valores de las concen- 
traciones en todas las fracciones fueron todavía cien veces por encima del límite permisible. Estos resultados permiten establecer que el material estudiado es un residuo altamente peligroso.

Figura 1. Concentración de Mercurio en las fraccion de la prueba de lixiviación DIN S4 en incrementos de la razones L/S para las dos muestras de lodos mercualires

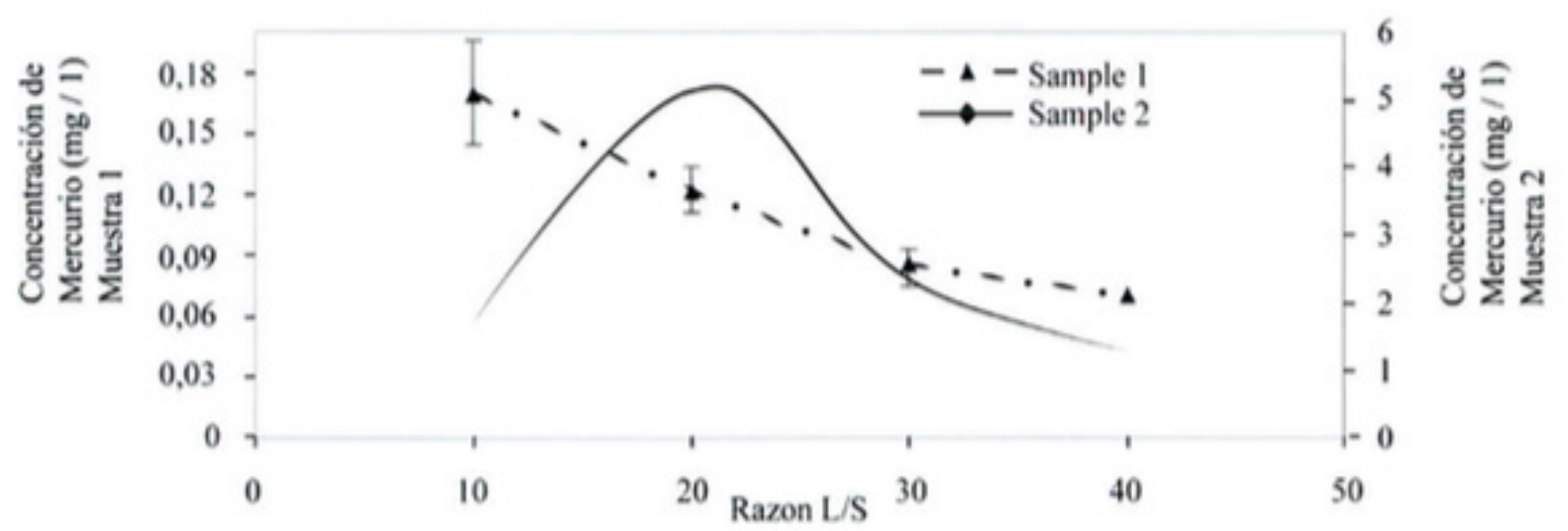

Fuente: autora.

Se desprende de los datos de la prueba de lixiviación que el tratamiento actual de los residuos de mercurio no es lo suficientemente eficaz para estabilizar el mercurio en dicha matriz. La lixiviación a largo plazo y la migración de contaminantes de los residuos dispuestos inadecuadamente pueden dar lugar a la contaminación de las aguas superficiales y subterráneas $(1 ., 1998)$. Por lo que son necesarios para evaluar la posible liberación de mercurio de la matriz de lodo realizar ensayos de lixiviación a largo plazo. Sin embargo, es bien sabido que la lixiviación y la biodisponibilidad de metales traza en suelos y sedimentos dependen de sus asociaciones químicas y físicas (N. B. J., 1991); (G., 1993); (N. M . L., 1997).

Los metales en suelos y sedimentos pueden formar fases minerales específicas, estar enlazados libremente en sitios intercambiables, co-precipitar, ser absorbidos en fases minerales, fijados por la materia orgánica y sulfuros o estar enlazados estructuralmente en las estructuras de alumino-silicato (Belzile N., 1989). El destino, transporte y la biodisponibilidad del mercurio en el lodo dependen de la especie en la que está presente (Liu G., 2006).

El sulfuro de mercurio $(\mathrm{HgS})$ es polimórfico, existente como el usualmente visto rojo $(\alpha)$, forma conocida como cinabrio y en forma metaestable color negro $(\beta)$, el metacinabrio. Ambas formas son muy insolubles en 
agua (Brandon N . P., 2001). El negro HgS (metacinabrio) se forma por la adición de H2S o Na2S a los iones acuosos $\mathrm{Hg} 2+$ y existe indefinidamente a temperatura ambiente sin formación de cinabrio (Brandon N. P., 2001). La estabilización de los lodos de mercurio en la industria cubana utilizando el Na2S pretende convertir el mercurio en $\mathrm{HgS}$ insoluble.

Las elevadas concentraciones de $\mathrm{Hg}$ observadas en las fracciones del ensayo DIN, especialmente para la muestra 2, indican la presencia de formas de mercurio más solubles. El HgC12 tiene una solubilidad práctica en agua de $70 \mathrm{~g} / \mathrm{l}$. (Bollen A., 2008) informó de que una alta concentración de mercurio en el lixiviado de los suelos contaminados se debe principalmente a la presencia de $\mathrm{HgC12}$. Sin embargo, también la solubilidad de las formas de mercurio sulfurado puede ser mayor de lo esperado por las extremadamente bajas constantes de solubilidad de los compuestos, teniendo en cuenta el alto $\mathrm{pH}$ de los lodos mercuriales. A pH alto, un aumento de la solubilidad de $\mathrm{HgS}$ resulta de la formación de formas de mercurio polisulfuro como $\mathrm{HgS} 22-\mathrm{y} \mathrm{Hg}(\mathrm{Sx}) 22$ (con Sx2- x=3-6) (R. P. K., 1997); (Jay J. A., 2000); (Svensson M., 2006). Además, la oxidación de sulfuros de mercurio durante el proceso de lixiviación puede contribuir a mejorar la movilidad de mercurio (Barnett $\mathrm{M}$. 0., 2001); (Holley E: A., 2007).

\section{Conclusiones}

Los resultados de la prueba de lixiviación muestran claramente que el proceso de estabilización aplicado a los lodos mercuriales no es capaz de prevenir la lixiviación significativa de mercurio. Sitios de disposición históricos pueden no cumplir con los estrictos requisitos necesarios para garantizar el almacenamiento seguro de este material de desecho altamente peligroso. En nuestro caso de estudio, las medidas correctivas son definitivamente necesarias para evitar una mayor dispersión del mercurio presente en lodos para eliminar el mercurio o reducir la lixiviación a niveles aceptables.

Alternativamente, es posible que el material sea dispuesto en los sitios de eliminación dedicados para ellos, y estén herméticamente sellados y completamente controlados. Mejores técnicas para estabilizar los lodos mercuriales generados también pueden contribuir a un manejo seguro y almacenamiento de los residuos. Se necesita más investigación sobre la lixiviación a largo plazo, la especiación del mercurio y de las tecnologías de tratamiento de desarrollar estrategias adecuadas para la gestión de estos residuos.

Afortunadamente, la tendencia a desplazarse hacia otras tecnologías, que no requieran mercurio disminuirá su uso, y eventualmente conllevará a eliminar la producción de estos lodos residuales mercuriales altamente peligrosos. 


\section{Bibliografía}

2008/1/EC, D. (2008). Directiva 2008/1/EC of the European Parliament and the Council of 15 January 2008 concerning integrated pollution prevention and control. Retrieved from http://europa.eu/legislation_summaries/environment/waste_management/128045en.htm

38414-S4, D. (1984). Schlamm und Sedimente, Bestimmung der Eluierbarkeit mit Wasser. DIN Deutches Institut Für Normung. Berlín.

91/689/EEC, D. (1991). Directiva of the European Parliament and the Council of 12 December 1991 concermng on hazardous waste. Retrieved from http://eurlex.europa. eu/LexUriServ/LexUriServ.do?uri=CELEX:31991L0689:EN :HTML

Barnett M. O., T. R. (2001). Oxidative disolution of metacinnabar ( $\beta$-HgS) by dissolved oxygen. Applied Geochemistry, $1499-1512$.

Bayar S., D. 1. (2009). Modelling leaching behaviour of solidified wastes using back propagation neural networks. Ecotoxicology and Environmental Safety, 843-850.

Belzile N., L. N. (1989). Heavy metal extractability in long term sewage sludge amenes soils.Environmental Science and Tecnology, 1015-1020

Biester H., M. G. ( 2002) : Estimating distribution and retention of mercury in three different soils contaminated by emissions from chlor-alkali plants: part 1. Science of the Total Environment, 177-189.

Bollen A ., W. A . (2008) . Mercury speciation in $\mathrm{HgC12}$-contaminated soils and ground water-Implications for risk assessment and chemical strategies. Water Research , 91-100.

Brandon N. P., F. P. (2001). Thermodyna mics and electrochemical behavior of Hg-S-Cl-H20 systems. Journal of Electroanalyti cal Chemistry, 18-32.

C., B . (2004). Muestreo de suelos. Criterios básicos. Revista Patagonia Forestal, 9-12.

Cottenie A. , V. M. (1982). Chemical analysis of plant and soil. Brussel: IWONL.

E., A. L. (1998). Diatomite. U .S. Geologi cal Survey Mineral Commodity Summaries, 56-57.

E., N . R . (1982). Carbonate and gypsum. In R. M. A.L. Page, Methods of Soil Analysis, Part 2. Chemical and Microbiological Properties, Chemical and Microbiological Properties (pp. 181-197). Madison: WI: E Publishing Inc.

Ecology, W. S. (2003). An assessment of laboratory leaching tests for predicting the impacts of fill material on ground water and surface water quality. Retrieved from: http//:www.ecy.wa.gov/programs/tcp/cleanup.html

Fytianos K., C. E. (1998). Leaching ofw heavy metals from municipal sewage sludge. Environment International, 467-475. 
G., T. F. (1993). Leaching behavior and fractionation of selected metals remediation as affected by thermal treatment of polluted sediments. International Journal of Environmental Analytical Chemistry, 167-175.

Gonzalez-Fernandez O., M. E. (2009).Multielemental analysis of dried residue from metal-bearing waters by wave length dispersive X-ray ftuorescence spectrometry. Spectrochimica Acta B, 184-190.

H., K. P. A. (1984). Trace elements in soils and plants. New York, US: CRC Press, Inc.

Holley E. A., M. A. (2007). Mercury mobi lization by oxidative dissolution of cinnabar (a-HgS) and metacinnabar (-HgS). Chemical Geology, 313-325.

I., M. J. (1998). Chemical partitioning of heavy metals in soil rock at historical lead smelter site. Water, Air and Soil Pollution, $391-409$.

Jay J. A., M. F. (2000). Mercury speciation in the presence of polysulfides. Environmen tal Science and Technology, 2196-2200.

K., M. S. (2009). Hidden Costs: Reduced IQ from Chlor-Alkali Plant Mercury Emissions Harms the Economy. Retrieved from: http:// na.oceana.org/en/news-media/publications/reports/hidencosts-reduced-iq-from-chlor-alkali-plantmercury-emissions-harms-the-economy

Kazi T. G., J. M. (2005). Evaluating the mobility of toxic metals in untreated industrial wastewater sludge using a BCR sequential extraction procedure and a leaching test. Analytical and Bioanalytical Chemistry .

Kylefors K., A. L. (2003). A comparison of small-scale, pilot-scale and large-scale tests for predicting leaching bahaviour of landfilled wastes. Waste Management, 45-59.

Landis M. S., K. G.-W. (2004). Divalent in organic reactive gaseous mercury emissions from a mercury cell chlor-alkali plant and its impact on near-field atmospheric dry deposition . Atmospheric Environment.

Liu G., C. J. (2006). Mercury characterization in a soil sample collected nearby the DOE Oak Ridge Reservation utilizing sequential extraction and thermal desorption method. Science of the Total Environment, 384-392 .

M., U. A. (1990). Methods of analysis for heavy metals in soils. In B. J. Alloway, Heavy Metals in Soils. (pp. 40 -73). Glasgow: Blac kie and Son.

Mukherjee A. B., Z. R. (2004). Mercury in waste in the European Union: sources, disposal methods and risks. Resources, Conserva tion and Recycling, 155-182.

N ., B. J. (1991). Impact ofheavy metals on low land rivers and implications for the man and the environment. Science of the Total Environment, 207-258.

N., M. L. (1997). Chemical fractionation of cadmium, copper, nickel and zinc in contaminated soils. Journal of Environmental Quality, 259-264 
Pacyna E. G., P. J.-J. (2006). Mercury emissions to the atmosphere from anthropogenic sources in Europe in 2000 and their scenarios until 2020. Science ofthe Total Environment, 147-156.

PNUMA. (2009). Global Estimate of Global Mercury Cell Chlorine Capacity. Retrieved from www.unep.org: http://www.unep.org/ hazardoussubstances/Mercury/PrioritiesforAction/ChloralkaliSector/Reports/tabid/4495/language/enUS/Default.aspx

Quevauviller P. H., V. d. (1996). Conclusions of the workshop: harmonization of leaching/extraction tests for environmental risk assessment. Science of the Total Environment, 133-139.

R., C. V. (2008). The use of leaching tests to study the potential mobilization of heavy metals from soils and sediments : A comparison. Water, Air and Soil Pollution, 95-11.

R., P. K . (1997). Inorganic Speciation of mercury in sulfidic waters : the importance of zero-valent sulfur. Environmental Science and Technology, 2148-2153.

Reis A. T., M. S. (2009). Mercury contamination in the vicinity of a chlor-alkali plant and potential risks to local population. Science of the Total Environment , 2689-2700.

Rhoades J., K. A. (1992). The use of saline waters for crop production - FAO irrigation and drainage. Rome , Italy.

S., R. D (1996). Recommended methods for determinig soil cation exchange capacityty. In A. W. J.T. Sims, Recommended soil tes ting procedures for the North-eastern United States (pp. 62-69). United States: Delaware: E-Publishing Inc.

Southworth G. R., L. S. (2004). Fugitive mercury emissions from a chlor-alkali factory: sources and fluxes to the atmosphere. Atmospheric Environment, 597-611.

Svensson M., D. A. (2006). Formation of cinnabar-estimation of favourable conditions in a proposed Swedish repository. Journal of Hazardous Materials, 830-836.

Ulrich S., 1. M. (2007). Mercury contami nation in the vicinity of a derelict chlor-alkali plant. Part 1: sediment and water contamination of Lake Balkyldak and the River Irtysh. Science of the Total Environment, 1-16.

USEPA. (2008). Land Disposal Restrictions Regulations for Mercury-Containing Non wastewaters. R 40 CFR Part 273 Retrieved from http ://www.epa.gov/epawaste/hazard/tsd/mercury/treatmnt.htm.

Van Ranst E., V. M. (1999). Manual for the Soil Chemistry and Fertility Laboratory. Ghent, Belgium: Ghent University, Faculty for Agricultural and Applied Biological Sciences.

Viguri J., A. A . (2000). Characterization of metal finishing sludge : influence of the pH. Journal of Hazardous Materials, 63-75. "Se- 
